Veritas Et Scientia

Vol. 7, N², 970-978

Julio - Diciembre del 2018.

ISSN Edición Online: 2617-0639

https://doi.org/10.47796/ves.v7i2.74

\title{
ANÁLISIS COMPARATIVO DE EDIFICIOS CON SISTEMAS ESTRUCTURALES DE CONCRETO ARMADO: "DUALES VERSUS DIAGRID".
}

\author{
COMPARATIVE ANALYSIS OF BUILDINGS WITH ARMED CONCRETE STRUCTURAL SYSTEMS: \\ "DUALES VERSUS DIAGRID".
}

\author{
José Luis Núñez Herrera ${ }^{1}$ \\ Presentado: $14 / 07 / 2018$ \\ Aceptado: $13 / 08 / 2018$ \\ Publicado online:27/12/2018
}

\section{RESUMEN}

Con la finalidad de investigar el comportamiento sísmico, de las estructuras Diagrid de concreto, se plantea 03 modelos de edificios de 06 niveles con una planta típica de $30 \mathrm{~m} \times 37.5 \mathrm{~m}$ ubicado en una zona de alta sismicidad en el Perú. Se ha realizado un análisis comparativo entre dos sistemas estructurales por un lado el Sistema Convencional u ortogonal y por el otro el sistema de Diagrid de Concreto armado, para este último se analizarán dos tipologías o modelos que denominaremos tipo $A$ y tipo $B$, cuya modulación será cada 02 y 04 pisos respectivamente. Los tres modelos tendrán las mismas características; área techada, dimensiones en planta y elevación, materiales de construcción y solicitaciones de cargas, de ubicación geográfica, tipo de suelo, uso e importancia, Coeficiente Básico de Reducción de la Fuerza Sísmica (Ro), estas características generan los factores que están normados por la E.030.2016 que define el procedimiento para el análisis sísmico. En esta Investigación se ha demostrado que de los Edificios estudiado (03 modelos) los que son con el Sistema estructural Diagrid de concreto armado (Diagrid tipo A y Diagrid tipo B) son más eficientes que el Sistema convencional (pórticos con muros estructurales). El peso de la estructura redunda en menores costos y menores demandas de estructuras de cimentación de los tres modelos. El más liviano es el Sistema Diagrid tipo B. Ambos sistemas Diagrid son el 30\% más livianos que el sistema convencional. La cantidad de acero necesaria para las diagonales de los sistemas Diagrid tipo A es $29 \%$ menos que el acero longitudinal necesarios para los muros estructurales del sistema convencional, mientras que Diagrid tipo B, requiere $40 \%$ menos que el sistema convencional.

Palabras clave: Diagrid, Sistema convencional, zona de alta sismicidad, Coeficiente básico de Reducción de la fuerza sísmica.

\footnotetext{
${ }^{1}$ Magister en Ingeniería Civil con mención en Estructuras
} 


\begin{abstract}
In order to investigate the seismic behavior of the concrete Diagrid structures, 03 models of 06-level buildings are proposed with a typical $30 \mathrm{~m}$ x $37.5 \mathrm{~m}$ plant located in an area of high seismicity in Peru. A comparative analysis has been made between two structural systems on the one hand the conventional or orthogonal system and on the other the Diagrid system of reinforced concrete, for the latter two types or models that we will call type $A$ and type $B$, whose modulation will be analyzed, will be analyzed. every 02 and 04 floors respectively. The three models will have the same characteristics; roofed area, dimensions in plan and elevation, construction materials and loading loads, geographical location, soil type, use and importance, Basic Coefficient of Reduction of the Seismic Force (Ro), these characteristics generate the factors that are regulated by E.030.2016 that defines the procedure for seismic analysis. In this research it has been shown that of the buildings studied (03 models) those with the structural Diagrid system of reinforced concrete (Diagrid type A and Diagrid type B) are more efficient than the conventional system (frames with structural walls), weight of the structure results in lower costs and lower demands of foundation structures of the three models, the lightest is the Diagrid type B system, both Diagrid systems are $30 \%$ lighter than the conventional system, the amount of steel needed to the diagonals of the Diagrid type A systems is $29 \%$ less than the longitudinal steel required for the structural walls of the conventional system, while Diagrid type B, requires $40 \%$ less than the conventional system.
\end{abstract}

Key words: Diagrid, conventional system, zone of high seismicity, basic coefficient of reduction of seismic force.

\title{
INTRODUCCIÓN
}

Desde el año 2000 se aprecia una clara tendencia a preferir las estructuras Diagrid en los países desarrollados por haber demostrado ser más eficientes estructuralmente, con elegancia arquitectónica y altamente adaptables a una amplia gama de formas en edificios (rectangulares, circulares, esferas, semiesferas, cilíndricas, formas combinadas, etc.). El término "Diagrid" es una mezcla de las palabras "diagonal" y "grid" (cuadricula) y se refiere a un sistema estructural que se basa en una cuadricula en elevación con ángulos diversos (no ortogonales) y gana su integridad estructural mediante el uso de la triangulación. Los sistemas de Diagrid pueden ser planos, cristalinos o adoptar múltiples curvaturas; a menudo usan formas curvas para aumentar su rigidez. Los pocos edificios de Diagrid de concreto armado que existen, para lograr su rentabilidad frente a otras estructuras tradicionales, han usado elementos de concreto postesado en las diagonales y losas, con esto dieron solución a la falta de capacidad de tracción del concreto, por consiguiente, se tiene edificios Diagrid de concreto económicos con un ahorro de concreto y acero. Las edificaciones en el Perú de más 06 pisos son en su gran mayoría diseñados con sistemas estructurales de concreto armado con el 
sistema Dual alcanzando la altura de hasta 200 metros (60 pisos); sin embargo, a mayor altura más grandes son las placas para rigidizar la estructura, conllevando a la disminución de las áreas aprovechables y la disminución de la transparencia de su perímetro y el incremento del costo de las estructuras. En el Perú no se han publicado trabajos de investigación sobre estructuras tipo Diagrid de concreto, por eso el Reglamento nacional de edificaciones vigente, no ha normado este sistema estructural.

Sepideh Korsavi et al. refiere que edificios de Diagrid han sido capaces de abordar la mayoría de los requisitos de diseño. Mingze Sun afirma que, en los últimos años, Sistemas de Diagrid de concreto han sido más ampliamente utilizado en los diseños actuales de construcción especialmente en edificios de mediana altura. Probaron que estas construcciones eran más rígidas y más eficaces en las restricciones de las derivas, pero menos satisfactoria en la limitación de las máximas aceleraciones en comparación con las edificaciones con estabilizadores. Sun concluye que los modelos con Diagrid de concretos tuvieron una menor aceleración en comparación con Diagrid de acero, porque Diagrid de concreto tiene períodos naturales más grandes. María Merlano llegó a la conclusión que los pórticos arriostrados, tienen un mejor comportamiento para edificios altos, ante fuerzas sísmicas presentando menores desplazamientos, menores cortantes y momentos de volcamiento y requiere menores cantidades de materiales, que los otros modelos analizados.

Arturo Quiroz en el 2016, afirman que el uso de rejillas rígidas ha resultado ser una solución para estructurar de manera eficiente edificios altos alrededor del mundo. Rohit Kumar indica que recientemente el sistema estructural de Diagrid ha sido ampliamente utilizado para edificios altos debido a la eficacia estructural y estética proporcionado por la configuración geométrica única del sistema. Afirma que la estructura Diagrid es económico en comparación con estructuras convencionales. Como una característica estructural cada vez más popular en los diseños actuales de construcción, "Diagrid" está bien definido como una mezcla de "diagonal" y "rejilla" para representar la configuración distribuida uniforme y triangulada (Boake, 2013).

\section{OBJETIVOS}

Comparar la respuesta sísmica de edificios de seis pisos con el sistema Dual de concreto armado y la respuesta sísmica de edificios de seis pisos con el sistema Diagrid de concreto armado, con los parámetros, categoría $C$ de nuestra norma, en la zona 4 y con el perfil de suelo tipo $\$ 2$ en edificaciones de 6 niveles.

\section{METODOLOGÍA}

Según la finalidad, se ha realizado una Investigación básica. Se fundamentó en un argumento teórico y su intención fundamental consiste en verificar el comportamiento sismo resistente de edificaciones de diferentes sistemas estructurales tipo Diagrid y sistemas tradicionales ortogonales, ambos de concreto armado. Se trabajó con un proyecto de edificación de seis niveles prototipo para uso $C$ de planta típica y regular. Cálculo del análisis modal con el programa ETABS. Se define la función espectral de acuerdo a los parámetros de la norma E.030. Se realizó un análisis comparativo del comportamiento sismo resistente entre de edificaciones de diferentes sistemas estructurales tipo Diagrid y sistemas Duales. El prototipo de edificación es aplicable a la costa peruana que por su cercanía con la concurrencia entre las placas de nazca y continental presenta mayor intensidad y frecuencia de movimientos 
sísmicos, el perfil de suelo seleccionado fue de tipo S2 (suelos intermedios). Se utilizaron los esquemas AutoCAD, para el modelamiento cálculo y obtención de resultados ETABS.

\section{RESULTADOS}

Se realizó un análisis comparativo entre dos sistemas estructurales por un lado el denominado Sistema Convencional u ortogonal que tiene elementos verticales (Placas y Columnas) y horizontales (vigas y losas diafragma) y por el otro un sistema no aun no usado en nuestro País que se denomina Sistema de Diagrid de Concreto armado, para este se analizarán dos tipos o modelos que denominaremos tipo A y tipo B cuya modulación será cada 02 y 04 pisos respectivamente.

\section{COMPARACION ENTRE EL SISTEMA CONVENCIONAL (ORTOGONAL) Y EL DIAGRID DE CONCRETO ARMADO}

\section{Análisis Comparativo de los 03 Modelos}

Para poder evaluar el comportamiento se presenta tres modelos, con las mismas características de funcionalidad arquitectónicas:

- Área techada total del edificio: $6750 \mathrm{~m} 2$

- Área del Lote: $1125 \mathrm{~m} 2$

- Número de piso: 06

- 7 ejes sentido $X$ con una Long entre ejes de $5 \mathrm{~m}$

- 6 ejes sentido $Y$ con una Long entre ejes de $7.5 \mathrm{~m}$

El material empleado para los tres modelos es el concreto armado con una resistencia a la compresión de $280 \mathrm{~kg} / \mathrm{cm} 2$, las vigas y losas aligeradas planteadas son las mismas en los tres modelos. Las exigencias propias del lugar donde se construirían y el uso son el mismo para los tres modelos.

Las diferencias entre el planteamiento estructural de los tres modelos es la siguiente:

\begin{tabular}{|l|l|}
\hline Tipo & Sistema Estructural \\
\hline Convencional & Dual de Concreto armado \\
\hline Diagrid tipo A (cada dos pisos) & Diagrid de Concreto armado \\
\hline Diagrid tipo B (cuatro pisos) & Diagrid de Concreto armado \\
\hline
\end{tabular}

Fuerzas internas en los muros y diagonales 


\begin{tabular}{|l|r|r|r|}
\hline Sistemas: & convencional & \multicolumn{1}{|c|}{ tipo A } & \multicolumn{1}{c|}{ tipo B } \\
\hline Fuerza cortante & $1,092.00$ & 84 & 58.24 \\
\hline Momento flect & $13,986.36$ & 48.00 & 96.00 \\
\hline
\end{tabular}

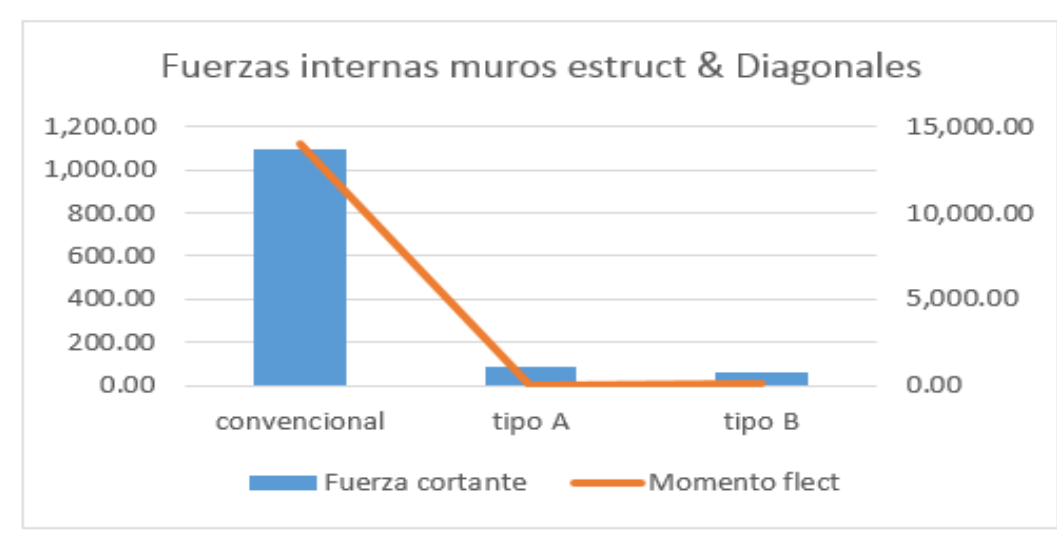

Figura

01: Fuerzas internas en los muros y diagonales

En el sistema convencional compuesto por pórticos o muros estructurales las fuerzas sísmicas producen fuerzas internas de cortante y momento flector, mientras en los sistemas estructurales Diagrid se manifiestan en las fuerzas axiales. En la figura 01, podemos apreciar que para las fuerzas cortantes en las diagonales no son ni el $8 \%$ de las fuerzas cortantes en los muros estructurales. De manera similar, pero con mayor diferencia se da con los momentos flectores donde en las diagonales no son ni el $0.7 \%$ de los momentos que se producen en los muros estructurales.

Existen muchas variables que determinan el costo de la edificación, pero en el sistema estructural tipo Diagrid tipo " $A$ " es más liviano y requiere menos concreto en un $28 \%$. El Sistema estructural tipo Diagrid tipo "B" es más liviano y requiere menos concreto en un $30 \%$, que el sistema convencional. La cantidad de acero longitudinal del acero de refuerzo necesaria para la Diagrid tipo A es $29 \%$ menos que el acero longitudinal del acero de refuerzo de las placas y columnas del sistema convencional. El Diagrid tipo B requiere $40 \%$ menos de acero longitudinal que en el sistema convencional. El acero de refuerzo transversal, está relacionado directamente con los esfuerzos cortantes, que en sistemas convencionales solicitan concentración de estribos en los extremos de las columnas y distribuido en las placas; en los sistemas Diagrid los cortantes no son significativos, ya que la fuerza sísmica es absorbida por las fuerzas axiales de compresión y tracción de las diagonales, por lo que adicionalmente a lo indicado en el párrafo precedente se debe tener un ahorro de acero de refuerzo transversal en los sistemas Diagrid.

Respecto a la eficiencia estructural, para cuantificarla en los 03 modelos comparamos las siguientes variables: Periodo de vibración de la estructura por análisis modal y el desplazamiento relativo inelástico o corregido [Deriva (\%)

En el ítem 3 (Tabla 01), podemos ver la similitud de los valores entre el modo1 y el modo2. Esto es conveniente para la eficiencia estructural ya que implica una estructuración más eficiente disminuyendo la torsión en planta, el modo 3 está por debajo del $80 \%$ del modo 1 .

En el ítem 1 de la tabla 02 , se evidencia que la rotación se presenta primero que la traslación en $\mathrm{Y}$, esto no es conveniente para la eficiencia estructural ya que implica una falta de rigidez en 
el eje $X$ en comparación del eje $Y$, esto se debe a que se tienen dos muros estructurales en $X$, mientras que en $Y$ se tienen 04 .

En la tabla 03 se expone los periodos en sus tres primeros modos, el Sistema Diagrid tipo B es el que mejor se comporta, por dos razones; primero porque tiene los menores periodos de vibración y segundo porque sus dos primeros modos son similares.

Periodos y Formas de Modo

Tabla 01: Periodos Naturales

\begin{tabular}{ccrrr}
\hline ítem & Sistema & Modo1 & $\begin{array}{c}\text { Periodo } \\
\text { Modo2 }\end{array}$ & Modo3 \\
\hline 1 & Convencional & 0.476 & 0.451 & 0.445 \\
2 & Diagrid tipo A & 0.435 & 0.308 & 0.249 \\
3 & Diagrid tipo B & 0.344 & 0.326 & 0.274 \\
\hline
\end{tabular}

Tablas 02: Formas de Modo

\begin{tabular}{cccll}
\hline ítem & Sistema & Modo1 & \multicolumn{1}{c}{$\begin{array}{c}\text { Meriodo } \\
\text { Modo2 }\end{array}$} & \multicolumn{1}{c}{ Modo3 } \\
\hline & & & & traslación \\
1 & Convencional & traslación $X$ & rotación & $Y$ \\
2 & Diagrid tipo $A$ & traslación $Y$ & traslación $X$ & rotación \\
3 & Diagrid tipo $B$ & traslación $Y$ & traslación $X$ & rotación \\
\hline
\end{tabular}

Tabla 03: Periodo para las formas de modo

\begin{tabular}{ccrrr}
\hline ítem & Sistema/Modo & \multicolumn{3}{c}{ Periodo } \\
\hline & & traslación X & \multicolumn{1}{c}{ traslación $\mathbf{Y}$} & \multicolumn{1}{c}{ rotación } \\
\hline 1 & Convencional & 0.476 & 0.445 & 0.451 \\
2 & Diagrid tipo A & 0.308 & 0.435 & 0.249 \\
3 & Diagrid tipo B & 0.326 & 0.344 & 0.274 \\
\hline
\end{tabular}

Los periodos de vibración de las estructuras Diagrid son menores a los periodos de las estructuras convencionales, son más notorias en la traslación en el sentido de $\mathrm{X}$ donde el Sistema Diagrid es $35 \%$ menos que el sistema convencional y en la rotación el Sistema Diagrid es $45 \%$ menos que el sistema convencional. En la traslación en el sentido Y El sistema Diagrid tipo " $A$ " con el sistema convencional no hay diferencia significativa, esto se debe a que solo tiene 03 ejes rígidos, mientras que el Diagrid tipo "B" tiene 04 ejes por lo que este sistema es $23 \%$ menor que el sistema convencional.

En la figura 02 muestran los valores que en el sentido del eje $X$ evidenciando que el Sistema Diagrid tipo A y B controlan mucho mejor los desplazamientos que el sistema convencional siendo sus desplazamientos en ultimo nivel la mitad que en el sistema convencional. 
En la figura 03 muestran los valores que en el sentido del eje $Y$, quedando claro que el Sistema Diagrid tipo B controla mucho mejor los desplazamientos, que el sistema convencional, siendo sus desplazamientos en ultimo nivel el $60 \%$ que en el sistema convencional.

Desplazamientos

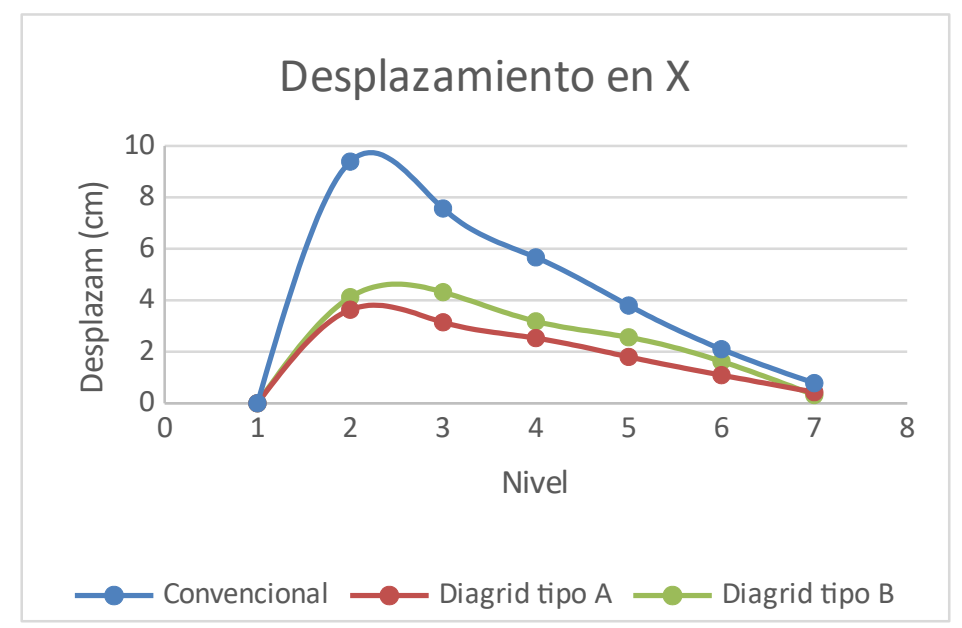

Figura 02: Desplazamiento en todos los niveles, en el sentido paralelo al eje $X$.

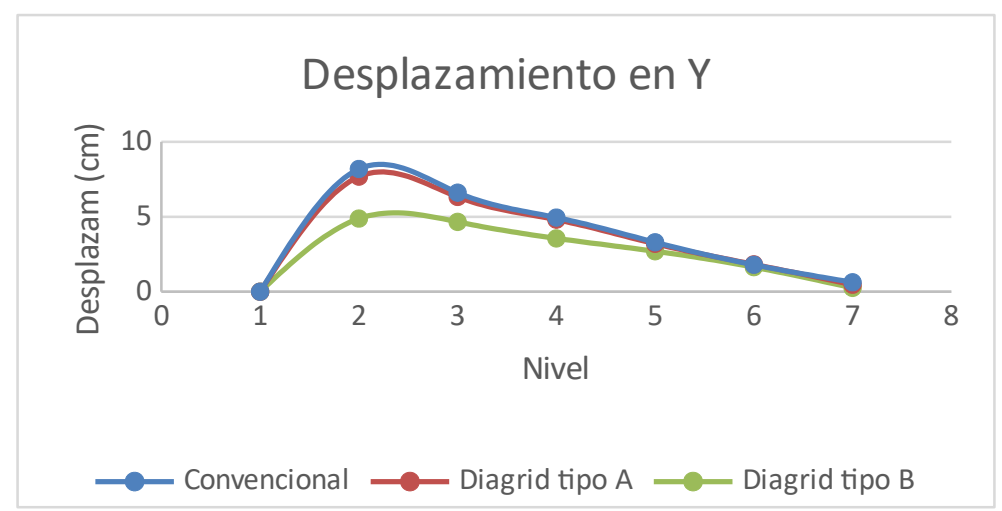

Figura 03: Desplazamiento en todos los niveles, en el sentido paralelo al eje Y.

En los desplazamientos o traslaciones las diferencias son contundentes entre los 03 modelos y son los siguientes:

$\checkmark \quad$ El Diagrid tipo A, se desplaza el 61\% del sistema convencional, en el sentido X y en el sentido $Y$ son similares.

$\checkmark$ El Diagrid tipo B, se desplaza aproximadamente la mitad que el sistema convencional, tanto en el sentido $\mathrm{X}$ como en $\mathrm{Y}$. 
Las Derivas en el sentido $X$, que presenta el sistema Diagrid (ambos modelos) son aproximadamente la mitad que el sistema convencional, mientras que en el sentido $Y$ la variación en sentido $Y$, es del $40 \%$ menos el sistema Diagrid que el convencional. Las curvas de desplazamientos y derivas presentan una curvatura fluctuante con tendencias de subida y bajada alternadas, esto se debe a las variaciones de secciones cada dos niveles.

El Sistema Diagrid de concreto armado, tanto en el modelo tipo A como en el tipo B es tiene menor periodo y menores desplazamientos que el modelo Convencional, destacándose el Modelo tipo B (con modulación cada 04 pisos) lo que representa menores daños ante sismos moderados por lo que podría ser una alternativa competitiva con otros sistemas como los aisladores sísmicos establecimientos de salud, donde los equipos médicos no deben alterarse en un evento sísmico, ni mucho menos dejar de funcionar.

\section{DISCUSIÓN}

En el Análisis estructural de los Edificios estudiados (03 modelos) dos con el Sistema estructural Diagrid de concreto armado (Diagrid tipo A y Diagrid tipo B) y uno con el Sistema convencional (pórticos con muros estructurales), se concluye que el sistema Diagrid de concreto armado es superior en los siguientes aspectos:

- Los Edificios Diagrid tienen un desplazamiento lateral y deriva menor que el sistema convencional.

- Son más livianos pesan $30 \%$ menos y se puede ahorrar entre el 30 y el $40 \%$ de acero de refuerzo, lo que implica menor costo del edificio.

- Son estructuras más dúctiles, ya que el mayor flujo de las cargas se da en forma axial y no por cortante que son las que producen fallas frágiles.

- Presenta más redundancia de elementos estructurales, esto sumado a la ductilidad podría representar un mejor comportamiento en el rango inelástico.

- En el aspecto estético y funcional tiene mayores posibilidades.

- Son más transparentes sus elevaciones, lo que permite mayor aprovechamiento visual e iluminación natural.

Una limitación del sistema Diagrid de concreto armado, es que solo se puede aplicar a edificios robustos donde la relación; ancho en planta entre altura total de la edificación, debe ser mayor que 0.7 , en edificios esbeltos (ratios mayores de 1.45) se pueden presentar fuerzas axiales en tracción muy altas, que superen el módulo de rotura del concreto, para ello se debe emplear materiales combinados como el caso de tubos de acero rellenos con concreto, como es el caso de los siguientes edificios: Guangzhou West Tower, Dorobanti Tower, entre otros. En busca de edificios más seguros, el desplazamiento lateral y deriva toma cada vez más importancia sobre todo en edificaciones Esenciales (Categoría A) y Edificaciones importantes (Categoría B). Los edificios Diagrid con una robustez entre 0.9 y 1.4 , tienen un mejor comportamiento que los edificios convencionales.

Es necesario que las Universidades y los investigadores, consideren realizar estudios relacionados con los sistemas Diagrid de concreto armado, como la capacidad del concreto armado de soportar fuerzas en tracción, determinar el rango del coeficiente Básico de Reducción Ro que correspondan con la capacidad real ductilidad, sobre resistencia y redundancia de las estructuras Diagrid. En la norma E.30 no se ha incluido el Sistema Diagrid ni sistemas arriostrados de concreto armado, inclusive aclara: "Estos coeficientes se aplicarán únicamente a estructuras en las que los elementos verticales y horizontales permitan la 
disipación de la energía manteniendo la estabilidad de la estructura". Los nudos en el sistema Diagrid, pueden presentar congestionamiento de acero de refuerzo, por lo que los Laboratorios de Estructuras de las Universidades, deben ensayar modelos de nudos, que permitan un sistema constructivo que garantice que el acero longitudinal mantenga el paralelismo axial, sin aglomerar de acero de refuerzo los nudos. Todos estos estudios e investigaciones serían remitidos a la comisión técnica del Reglamento Nacional de Edificaciones perteneciente al Ministerio de Vivienda, Construcción y Saneamiento.

En el mundo se evidencia por la clara tendencia de construir edificaciones más innovadoras, que la eficiencia de los sistemas Diagrid se va imponiéndose a los sistemas convencionales, sin embargo en nuestro País no existen investigaciones sobre Diagrid de; concreto armado, concreto postensados, Materiales Mixtos como los tubos de acero rellenos de concreto, estos estudios que serían de mucha utilidad para el desarrollo de infraestructura en nuestro país, previa incorporación en el Reglamento Nacional de Edificaciones.

\section{REFERENCIAS BIBLIOGRÁFICAS}

Allford Hall, Monaghan Morris. Documento informativo: "The Yellow Building Notting o el Edificio Amarillo" de Dale Village W11 en el Reino Unido, para uso de oficinas, con un área de $15,000 \mathrm{~m}^{2}$ en siete pisos.

Altamirano, Nicolas "Exposición del Edificio Diagrid para la televisión central de china CCTV"

Amador Terán, Gilmore et al 2016“Uso de rejillas perimetrales (Diagrid) para estructurar edificios altos de acero ubicados en zonas de alta sismicidad", Área de Sustentabilidad y Desarrollo Tecnológico en Ingeniería Civil, Universidad Autónoma Metropolitana, Azcapotzalco, México.

Arteta Torrentes, Carlos. Tesis de maestría "Alternativas de Rigidización en edificios altos en concreto para la zona de amenaza sísmica baja en la costa caribe Colombia." 2008 Universidad del Norte, División del Ingeniería, Maestría en Ingeniería Civil Barranquilla.

Khan, Ravish. "Analysis of diagrid structure in comparison with exterior braced frame structure". PG student, Department of Civil Engineering, Jawaharlal Nehru Engineering College, Aurangabad, Maharashtra, India.

Kumar Singh Rohit, Vivek Garg, Abhay Sharma." Analysis and Design of Concrete Diagrid Building and Its Comparison with Conventional Frame Building", Department of Civil Engineering, Maulana Azad National Institute of Technology, Bhopal, India.

Mccain, Ian. Exposition: "Diagrid Structural Efficiency \& Increasing Popularity".

Mele Elena, Toreno Maurizio. "Diagrid structures for tall buildings: case studies and design considerations". University of Naples Federico II, Faculty of Engineering, Department of Structural Engineering (DIST), Naples, Italy.

Meyer Boake, Terri. "Diagrid Structures; system, connection, details" School of Architecture, University of Waterloo, Cambridge, ON, Canada.

Panchal, Nishith. "Diagrid Structural System: strategies to reduce lateral forces on high-rise buildings". PG student, Applied Mechanics \& Structural

Quiroz Arturo Y Terán Amador et al. 2016 "Rejillas Rígidas en zonas de alta sismicidad: ventajas sismo resistentes y sustentables". Departamento de Materiales, Universidad Autónoma Metropolitana, Ciudad de México.

Sepideh Korsavi et al. 2014 "El proceso evolutivo de las estructuras Diagrid para Conceptos Arquitectónicos, estructurales y de sostenibilidad: revisión de casos de estudiados". 
Sun, Mingze et al.2007 "A comparative study on the seismic performance of concrete and steel Diagrid structures", Massachusetts Institute of Technology, Department of Civil and Environmental

Sun Moon, Kyoung et al. 2007 "Diagrid Structural Systems for tall buildings: characteristics and methodology for preliminary design"

NTE 030.2016, la norma técnica e.030 "Diseño Sismo resistente" del reglamento nacional de edificaciones, Ministerio de Vivienda Construcción y Saneamiento, Lima, Perú. 